\title{
Acceleration Response Characteristics of Body-conducted Sound Sensors; Comparison with Several Acceleration Sensors
}

\author{
Takeshi Joyashiki ${ }^{1,2}$, Chikamune Wada ${ }^{1}$ \\ ${ }^{1}$ Graduate School of Life Science and Systems Engineering, Kyusyu Institute of Technology \\ 2-4 Hibikino, Wakamatsu-ward, Kitakyushu-city, Japan \\ o899019t@edu.brain.kyutech.ac.jp; wada@brain.kyutech.ac.jp \\ ${ }^{2}$ Saiseikai Yahata General Hospital \\ 5-9-27 Harunomachi, Yahatahigasi-ward, kitakyusyu-city, Japan,
}

\section{Extended Abstract}

Lung sounds may be used for applications such as diagnosis [1] and biological information monitoring [2]. We focused on body-conducted sound sensors (BCS) [3] as sensors for lung sound detection. BCS were developed to detect non-audible murmurs (NAM) [4]. These devices can be used as bioacoustic sensors with high sensitivity and resistance to external noise. This study investigated the acceleration sensitivity of BCS of various shapes and masses using surface-free acceleration based on the hypothesis that such sensors should exhibit similar characteristics to the acceleration sensors of comparable structures.

Frequency characteristics were measured by simulation using the equivalent-circuit model. We produced systems for measuring the sensitivity of the systems and assessed the validity of the sensitivity measurements and responses to simulation. We compared BCS, which were characterized by a mass of $1.50 \mathrm{~g}$ and a contact area of $314 \mathrm{~mm}^{2}$, with accelerometers of various types, including BK: Type 8001 , with a sensitivity of $1 \mathrm{~V} / \mathrm{m} / \mathrm{s}^{2}$, mass of $30.89 \mathrm{~g}$, and contact area of $176.6 \mathrm{~mm}^{2}$ and Primo: S15S5C, with a sensitivity of $0.005 \mathrm{~V} / \mathrm{m} / \mathrm{s}^{2}$, mass of $0.28 \mathrm{~g}$, and contact area of $26.4 \mathrm{~mm}^{2}$. Each sensor was compared with the accelerometers using a sensitivity measurement system [5]. Evaluation was performed using a lung sound simulator. The simulator was constructed using parameters derived from published research [6]. A headphone was implemented inside the simulator and used the simulator as a sound source. An air chamber was installed and used to observe the reference sound source. A urethane elastomer simulating a body was placed in the chamber, and sensors were placed on it. The simulator was first tested with white noise and then evaluated by educational lung sounds. The lung sounds included normal trachea sounds and the polyphonic wheezes found in bronchial asthma.

The simulation results showed that sensitivity increases when a BCS has a small mass and a large contact area. Further, we produced systems for sensitivity measurement and assessed the validity of the sensitivity measurement and the simulation. The root-mean-square error of the result was 1.37-3.59 dB in a range of 100-2,000 Hz. However, in the 100-600 Hz range, peaks that were not reproduced by the simulation appeared in the measured values. Comparison of the results from each acceleration sensor showed that BCS had stable sensitivity due to an increase in frequency. The type 8001 exhibited decreasing sensitivity with increasing frequency. S15S5C exhibited stable sensitivity above $600 \mathrm{~Hz}$. Peak sensitivity was observed in each sensor at frequencies under $600 \mathrm{~Hz}$. White noise was similar to the frequency characteristics according to the sensitivity evaluation system in observations using the pulmonary sound simulator. BCS showed high sensitivity up to high frequencies, Type 8001 was highly sensitive in the low range, but in the high range the sensitivity decreased. The sensitivity of S15S5C on a whole was low. In the observation of lung sounds, normal tracheal sounds were observed over a wider band by the BCS, and wheezes in the high region were clearly confirmed.

We conclude that BCS have characteristics similar to those of acceleration sensors and that their frequency characteristics can be estimated by simulation in the 700-2000 Hz range. Additionally, high, stable sensitivity was obtained in the range of 600-2000 Hz. Through comparison with various acceleration sensors, we found that BCS is suitable for the measurement of bioacoustic sounds.

\section{References}

[1] T. Shimoda, Y. Obase, Y. Nagasaka, H. Nakano, R. Kishikawa, and T. Iwanaga, "Lung sound analysis can be an index of the control of bronchial asthma," Allergol Int, vol. 66, no. 1, pp. 64-69, 2017. 
[2] S.-H. Li, B.-S. Lin, C.-H. Tsai, C.-T. Yang, and B.-S. Lin, "Design of wearable breathing sound monitoring system for real-time wheeze detection," Sensors, vol. 17, no. 1, 2017.

[3] T. Hirahara et al., "Silent-speech enhancement using body-conducted vocal-tract resonance signals," Speech Communication, vol. 52, no. 4, pp. 301-313, 2010.

[4] Y. Nakajima, H. Kashioka, N. Campbell, And K. Shikano, "Non-audible murmur (NAM) recognition," IEICE TRANSACTIONS on Information and Systems, vol. E89-D, no. 1, pp. 1-8, 2006.

[5] T. Jouyashiki and C. Wada, "Estimation of the frequency response of body-conducted sound sensors via the equivalent circuit model(Japanese)," jsmbe, vol. 56, no. 2, pp. 44-51, 2018.

[6] S. S. Kraman, G. A. Pressler, H. Pasterkamp, and G. R. Wodicka, "Design, construction, and evaluation of a bioacoustic transducer testing (BATT) system for respiratory sounds," IEEE Trans Biomed Eng, vol. 53, no. 8, pp. 1711-1715, 2006. 